\title{
UAV Aided Data Collection for Wildlife Monitoring using Cache-enabled Mobile Ad-hoc Wireless Sensor Nodes
}

\author{
Umair B. Chaudhry, Chris I. Phillips \\ School of Electronic Engineering and Computer Science \\ Queen Mary University of London (QMUL), London, United Kingdom
}

\begin{abstract}
Unmanned aerial vehicle (UAV) assisted data collection is not a new concept and has been used in various mobile ad hoc networks. In this paper, we propose a caching assisted scheme alternative to routing in MANETs for the purpose of wildlife monitoring. Rather than deploying a routing protocol, data is collected and transported to and from a base station using a UAV. Although some literature exists on such an approach, we propose the use of intermediate caching between the mobile nodes and compare it to a baseline scenario where no caching is used. The paper puts forward our communication design where we have simulated the movement of multiple mobile sensor nodes in a field that move according to the Levy walk model imitating wildlife animal foraging and a UAV that makes regular trips across the field to collect data from them. The unmanned aerial vehicle can collect data not only from the current node it is communicating with but also data of other nodes that this node came into contact with. Simulations show that exchanging cached data is highly advantages as the drone can indirectly communicate with many more mobile nodes.
\end{abstract}

Keywords-UAV; caching; sensors; MANETs; WSN; waypoint

\section{INTRODUCTION}

The use of wireless sensor networks (WSNs) and mobile ad-hoc networks (MANETs) in various areas such as environmental monitoring, military, vehicular networks and animal tracking has been widely adopted [1]-[6]. Applications of such networks vary based on the targeted area. Humidity and seismic sensors, collision avoidance and parking sensors, pulse and temperature sensors are all examples of this. In WSNs, nodes are deployed with the intention of sensing and relaying information to a particular destination for evaluation purposes. In MANETs, nodes are mobile forming temporary networks throughout their runtime. Nodes in these networks are typically small and possess limited resources. They have restricted processing power and run on small batteries hence energy conservation is a serious concern for them. Data is routed from the source to the destination using routing protocols. The convergence and retransmission mechanisms of these protocols impose an additional overhead causing an additional energy drain. Many efforts have been put into making these protocols as efficient as possible [7]-[9]; however, there is always a trade-off. Conversely, we propose the use of an unmanned aerial vehicle (UAV) to periodically to collect data from caching assisted nodes, hence avoiding routing altogether.
In this paper, we focus on wildlife tracking and monitoring. Traditionally, this is achieved by strapping heavy tracking equipment to animals [10], [11]. Even with current technological trends, wildlife monitoring remains a challenging setting. Typical VHF transmitters are of very restricted range [12] and have a limited battery life and the ones that are longer in range are satellite oriented and hence require even more power, consequently providing a lower lifetime. Table I shows some of the existing devices available. Approaches such as [13], [14], [15], [16], [17] are either too expensive, require dedicated manpower, or the resource constraints of the devices can cause them to fail prematurely. Our aim is to make tracking and monitoring easier and less costly in terms of finance and operation.

Several tracking systems have already been proposed and some are even operational. ATLAS, in [16], employs 9 base stations and extensive computing to determine the location of flying animals. ARTS [14] uses VHF tags and hefty hardware and can only work on animals with small home ranges. [13], in addition to being composed of energy draining $3 \mathrm{G}$ modules, is highly dependent on manpower. [18] uses a three tier architecture and the authors claim energy conservation to be one of the biggest challenges of the model in addition to constraints such as the lack of physical intervention with the nodes after deployment and intermittent network connectivity. [15] uses dual chip collars and requires pre-processing at the nodes before transmission. Conversely, [19] requires image processing on static sensor nodes although the authors acknowledge that the accuracy of the system drops with time.

TABLE I. TRADITION DEVICES AND THEIR LIMITATIONS

\begin{tabular}{|l|l|l|l|l|}
\hline Devices & NANO & $\underline{\text { MICRO }}$ & $\underline{\text { SMALL }}$ & $\underline{\text { MEDIUM }}$ \\
\hline Weight Range & 5g-20g & 6g-50g & 20g-100g & $130 \mathrm{~g}-250 \mathrm{~g}$ \\
\hline $\begin{array}{l}\text { Example } \\
\text { Suitable } \\
\text { Animals }\end{array}$ & $\begin{array}{l}\text { Birds, } \\
\text { bats, and } \\
\text { other tiny } \\
\text { mammals }\end{array}$ & $\begin{array}{l}\text { Lizards, } \\
\text { tortoises, } \\
\text { turtles, frogs, } \\
\text { very small } \\
\text { mammals }\end{array}$ & $\begin{array}{l}\text { Animals } \\
\text { that weight } \\
\text { at least 500 } \\
\text { grams }\end{array}$ & $\begin{array}{l}\text { Foxes, } \\
\text { Tasmanian } \\
\text { Devils }\end{array}$ \\
\hline $\begin{array}{l}\text { Data } \\
\text { Recovery } \\
\text { Method }\end{array}$ & $\begin{array}{l}\text { UHF } \\
\text { Wireless }\end{array}$ & $\begin{array}{l}\text { UHF } \\
\text { Wireless }\end{array}$ & $\begin{array}{l}\text { UHF } \\
\text { Wireless }\end{array}$ & Satellite \\
\hline $\begin{array}{l}\text { Drone Data } \\
\text { Downloading }\end{array}$ & Standard & Standard & Standard & N/A \\
\hline $\begin{array}{l}\text { Base Station } \\
\text { Battery Life }\end{array}$ & 2 days & 5 days & 2 days & N/A \\
\hline
\end{tabular}


This paper highlights a routing-less approach for data collection from mobile sensor nodes for wildlife monitoring using an unmanned aerial vehicle. In our application scenario, we assume the home range to be a large field or area where the animals roam. Our sensor nodes, according to our use-case scenario, will be wildlife and several of these will be dispersed across the home range. The nodes are considered to be mobile with some degree of purpose in their movement but at the same time having some randomness in their behaviour. An unmanned aerial vehicle will make periodic trips across the field. The nodes are equipped with sensing equipment, the type of which is not the focus of our research. The nodes upon encountering the UAV transfer their data to it. In this paper, we test this approach in two scenarios. One is without communication between the nodes and the other with caching enabled among the nodes allowing them to store data from the nodes they come into contact with. Our simulator uses the Levy walk movement model for the nodes.

\section{RELATED WORK}

Retrieval of data from static and mobile sensor nodes deployed in a large field through an aerial vehicle is a fairly new concept. Lately, several researchers [24]-[36], have explored the use of a UAV to acquire data from sensor and adhoc nodes mainly due to the fact that a lot of energy draining forwarding and processing tasks are taken away from the resource sensitive nodes.

The authors in [20] describe their method of how a UAV can be used for wildlife monitoring and tracking. In their approach, the field is divided into virtual grids and each grid has a cluster of static sensors deployed. Each cluster has a cluster head which acts as a point of contact for the UAV to collect data. The network model is not a generic one and is highly dependent on data sets obtained from tracking equipment for specific animals. In their case, they used the movement data of zebras from ZEBRANET and the UAV visits the cluster heads of the most active grids for data collection. Another approach [21] has been to mount a cellular network base station on a UAV in an attempt to try to pick up cell phone data from users.

Another approach has been to mount a cellular network base station on a UAV in an attempt to try to pick up cell phone data from users [22]. While in communication with the nodes, the UAV allocates the whole bandwidth to the node with the least data in its buffer and moves towards it during the transfer. The drone moves with a moving node along the same trajectory and the base station may be in communication with more than one node at a time with different parameters. This requires it to adopt a consistent back and forth movement based on the attributes of the node(s) it is in communication with. Ignoring the back and forth movement, the synchronisation mechanism between the drone and a node may be considered suitable for cellular networks as the communication between the base station and the nodes may be extensive. However, the same cannot be said for mobile sensor nodes, especially when considering their limited resources.

Ariel assisted data collection using a UAV from limited capacity sensor nodes has also been considered in [25] using a Markov chain to model the movement of the UAV in addition to modelling the irregularities in the movement due to several implicit and explicit factors. The authors in [26] also favour the same concept of acquiring data from sensor nodes deployed in a field using a UAV. They add to the approach by proposing a model that allows the sensor nodes to cooperate with each other to achieve simultaneous transfer of data to the UAV to reduce latency. In addition, their work highlights how they can reduce packet losses occurring in the backward direction while the UAV is receiving data and moving forward using an efficient forwarding scheme. However, one can argue that cooperation between the nodes is of limited use as multiple nodes can transmit to the UAV simultaneously using different channels.

Considering the low data transfer rates due to the brief contact duration of a UAV with a sensor node in UAV aided data collection, the authors in [27] propose a modified Media Access Control (MAC) protocol which uses beacon broadcast at the UAV together with Carrier Sense Multiple Access/Collision Avoidance (CSMA/CA) at the ground nodes. The nodes have to contend with each other to speak to the UAV in addition to remaining in the listening mode to receive the beacon which is a big drawback considering the limited battery life of the nodes.

Other researchers have focused on the most optimal traversal path of the UAV in reference to an age element associated with the data picked up from the sensor nodes [28]. They define the age of information as the time data is sensed by the node to the time it is delivered to the sink by the UAV hence the flight path and duration of the UAV plays an important role. Theoretically, they show that the optimal route for the UAV corresponds to a Hamiltonian path and hence they propose a trajectory planning scheme accordingly. Nevertheless, their approach promotes the use of static nodes and dynamic programming which is computational complex.

The feasibility of using a UAV for data collection from ground sensors has been tested in [29] against several parameters including weather, flight height, latency, throughput, jitters and communication channels an authors have recommended a configuration based on their observations. However, it can be argued that the values are highly subjective.

The authors in [30] propose an automatic tracking system that offers autonomous wildlife monitoring. In their approach, they suggest to equip the wildlife with a system that is a combination of a Global Positioning System (GPS) module and a wireless Subscriber Identity Module (SIM). The GPS coordinates are sent to a central server which is also equipped with a SIM which forwards the information to a SIM-equipped control system from where the received information is fed to a drone in addition to the drone control commands. The drone, using this information and the control commands, navigates to the coordinate location. The information is fed to the drone only at the take-off point. Thus, once the drone reaches the target location, the target might not be there resulting in waste of time, energy and the trip. In addition, the devices used to accomplish this have a limited lifespan which is greatly affected by the presence of two highly energy draining modules on the animals. 
An approach that involves a combination of Q-learning and Neural Networks is considered in [31] to offer an energyefficient method for portable base station positioning using UAV. The authors use the concept of landing spots from [32] to move the UAV. Once a suitable position is achieved by the UAV in terms of connectivity, the UAV uses the landing spots to save energy by landing and providing coverage rather than continuing to hover.

In addition to the concept of using a UAV for data collection from wireless sensor network nodes, researchers have proposed BEE-DRONES [23] to achieve energy efficiency at the UAV level and at the network level. The UAV, as with pollinating bees, visits specific targeted ground sensors for data collection. Their work in addition to dealing with minimal path planning of the UAV targets the synchronization issues between the ground sensors and the drone and proposes a wakeup mechanism for the nodes in accordance to the expected visit time of the drone. An efficient path planning approach is also considered in [24], focusing on the limited flight time and energy constraint of the UAV for data gathering in WSNs. They also show how a cluster-head can be selected from a cluster of sensor nodes based on node energy and value of information. This cluster head is the contact point of the UAV for data collection. However, choosing a cluster-head is still an additional burden for the nodes, and data communication between the nodes and the cluster-head employs a traditional routing mechanism incurring protocol overhead.

The researchers in [33] promote the concept of using a UAV for data collection and offer an approach to make it feasible in terms of energy efficiency and security. They propose a centralized framework which allows the UAV to carry the remaining energy of the nodes to the sink which then decides which nodes are suitable candidates for the clusterhead and which nodes should be marked as compromised and hence disqualified from the cluster-head election. Centralizing the cluster-head selection can add an additional delay to the communication and can result in unwanted exchanges between the nodes and a cluster-head now labelled as a disqualified candidate. Also, the approach can cause severe fluctuation of the cluster-head assignment if the nodes are mobile.

Despite the preceding research studies, intermediate caching between the nodes has, to our knowledge, never been considered. We believe that intermediate caching between the nodes can be advantageous for several reasons. Firstly, it does not impose additional strain on a particular node (i.e. a clusterhead). Secondly, there are no limitations on the positioning of the nodes and, finally, the UAV has more flexibility in terms of points of contact.

The next section outlines our proposed system. This is followed in Section 4 with a simulation-based evaluation where we employ a Levy movement model for the mobile nodes together with four different variations of caching. Finally we conclude the paper in Section 5.

\section{SYSTEM MODEL}

We have implemented a discrete time event simulator in Java. Rather than feeding movement traces into the simulator
[20], our simulator has the ability to cope with different movement models, allowing for flexibility. However, for the sake of this paper, we only employ the Levy walk movement model. Historically, it was widely believed and accepted that animal movement could be explained by a simple random walk model; however, recent evidence has shown that the movement of different animal species are more relatable to the levy walk model [34], [35].

A Levy walk is based on the Levy foraging hypothesis that states that animals employ a Levy walk since it leads to optimal search efficiency. A Levy Walk according to Gautestad "describes a movement process with physical realism in the context of observing animal paths at fixed intervals" [36]. It is defined as a movement model where the subject continues to move in one particular direction and upon finding a resource patch, adopts random movement in and around it [37]. Fig. 1 from [38] highlights how a trace of a Levy walk may look.

Statistically, a Levy walk is a Markov based stochastic process. Rather than focusing on the biological nature of models, arguments over the past years have been restricted to statistical measures although the physicality and the movement pattern should matter more [39]. Humphries states "the real utility of the Levy research field is not, therefore, to accurately model animal movement paths, but as an exploratory tool to aid the behavioural analysis of animal movement datasets" [40]. Fig. 2 shows a typical Levy movement pattern of a node generated by our simulator.

In addition to the movement model, our model provides the opportunity for selectable caching depending on the scenario. In the case of Phase 1, we omit Intra-Node caching to provide baseline performance. In Phase 2 intra-node caching is enabled.

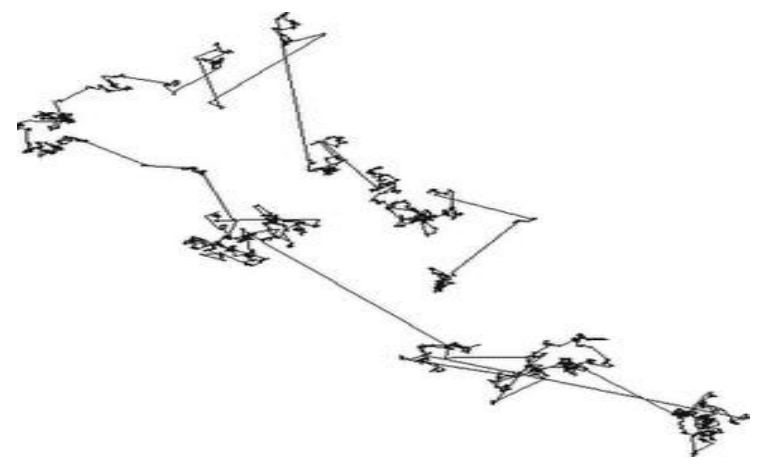

Fig. 1. Example Levy Walk [38].

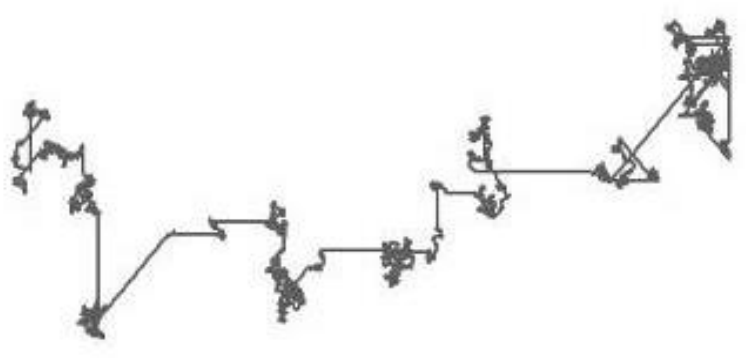

Fig. 2. Single Node Movement Trace for $12 \mathrm{hrs}$ at $11 \mathrm{kph}$. 


\section{A. Phase 1: No Intra-Node Caching}

In Phase 1, mobile nodes are dispersed in a field of size $\mathrm{n} \times$ $\mathrm{n}$. The nodes do not have any ability to cache data received from other nodes. In fact, the nodes do not even communicate with each other. Each node gathers/senses data from the host animal while the animal moves around. A UAV is to be sent out periodically from a gateway/base station and the UAV follows a fixed path based on waypoints [41], [42] which have been selected in such a way that the UAV can cover the majority of the field during its trip. In other words, the trajectory of the UAV is fixed, and the UAV moves from waypoint to waypoint as shown in Fig. 3.

Our model considers two versions of this approach. In the first (termed 'WP'), the UAV only captures the data cached at the designated waypoints and does not pick it up directly from the mobile nodes. These waypoints are equipped with caches and can cache date from the mobile nodes they have encountered. The UAV simply collects the data from the waypoint stations. This approach of capturing data from fixed waypoints imitates the existing approach of collecting data from static cluster heads in the field. In the second variation (termed 'UAV'), rather than collecting data from the waypoints, if during the trip, the UAV encounters any node that has data to transmit, the node transmits that data directly to the UAV. The UAV can either, store that data and bring it back to the base station at the end of the trip or can use some wireless technology to transmit that data to the base directly. In our approach, the UAV does not wirelessly transmit the data but brings it back to the sink at the end of its trip.

\section{B. Phase 2: Intra-Node Caching}

In this phase, in addition to everything described in Phase 1 , the nodes have an intra-node caching ability allowing them to cache data obtained during interactions with other mobile nodes they come into contact with. The caching mechanism can be better understood from Fig. 4.

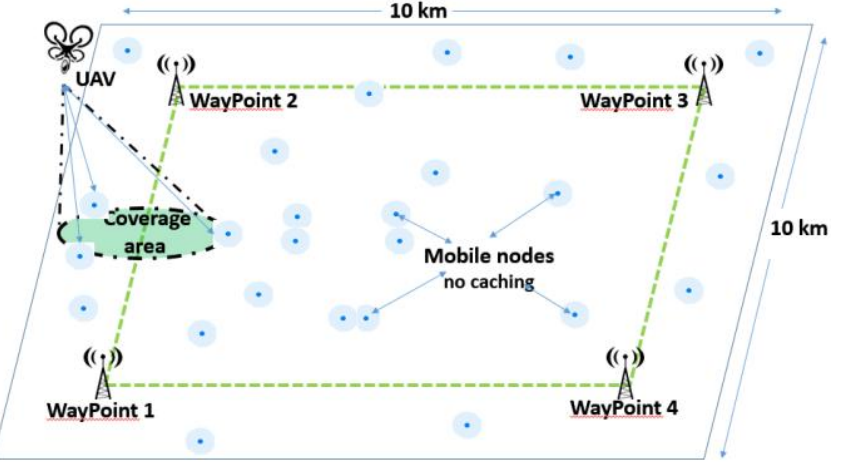

Fig. 3. Phase 1: No Intra-node Caching between Nodes.

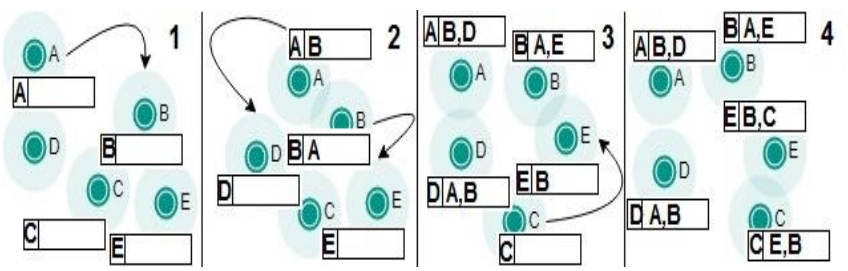

Fig. 4. Intra-node Caching Technique.
A node not only exchanges data with the node it comes in contact with directly but also exchanges data it has accumulated in its cache. Snapshot (2) of Fig. 4 shows that A and $\mathrm{B}$ exchange information upon coming in range of each other. Snapshot (3) shows that when D comes in contact with A, it not only caches A's information but in addition, caches all the information that $\mathrm{A}$ has in its cache (information $\mathrm{A}$ gathered from $B$ in its previous encounter with B). This means that even if a node has not encountered a certain node directly, it still has the ability to carry this "second-hand" data the node if it came into contact with it indirectly. Hence we refer to this as an 'indirect caching technique'.

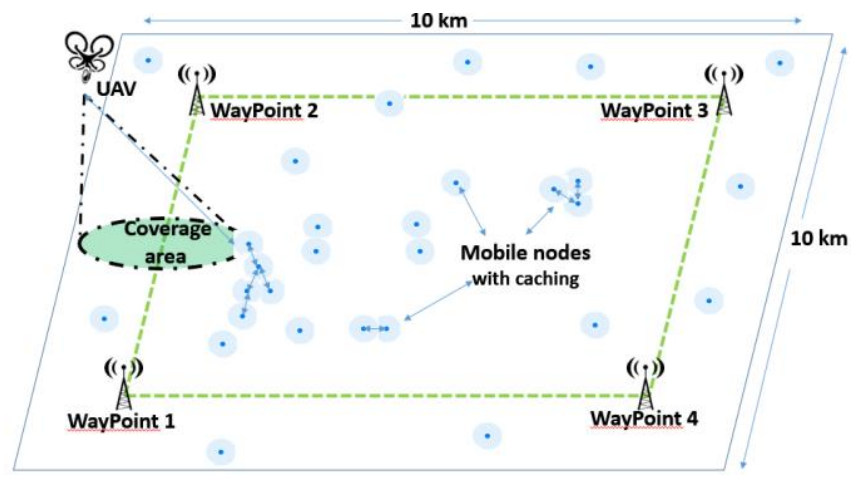

Fig. 5. Phase 2: Intra-node Caching between Nodes.

As shown in Fig. 5, the UAV follows the same path to complete its trip across the field which we term waypoint to waypoint communication. This phase also has two variants. The nodes have caching enabled in them in both versions which means if they come in contact with each other, they are allowed to exchange information and the receiving node can cache data that belongs to other nodes. In the first version (termed ' $\mathrm{WP}+\mathrm{C}$ '), the UAV only picks up data from the fixed route waypoints and not from the nodes directly. The fixed waypoints on encountering a node not only take the node's data but also accept all data that node has encountered from other nodes. In the second variant (termed ' $U A V+C$ '), if the UAV encounters a node that has data to send, the UAV collects that data directly. When the UAV encounters such a node, the node transfers to the UAV its own data in addition to the cached data of other nodes it has interacted with.

Data exchange between the nodes arises using a simple connectionless protocol shown as Algorithm 1. It assumes that nodes are transmitting regular beacons to announce their presence in the field in addition to scanning their coverage area to discover other nodes in their vicinity. This is the case with the UAV as well. If a node discovers another node in its vicinity (NeighborsFound $=1$ ), it exchanges its data along with all messages of other nodes in its cache to the encountered node. For the UAV, it does not exchange its data with the encountered node; however, it does pick up the other node's data in the same way. For intra-node communication, this is a two way process which means that both nodes in each other's vicinity will exchange information. A node upon receiving a message (DataToReceive $=1$ ) will first check its cache (NodeCache $=$ null) to see if it already has that data. If this is true, the node will discard the oldest version of the data (remove old message) replacing it with the newer version (push 
new message) by making use of timestamps (timestamps OldMsg

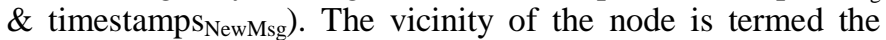
'range' of the node in this paper and is defined by ' $i$ ', the radius around the node and its coverage area. The range of the node is one of the design parameters that we consider in subsequent experiments. Using this protocol, we eliminate the possibility of having duplicate data on nodes at any time.

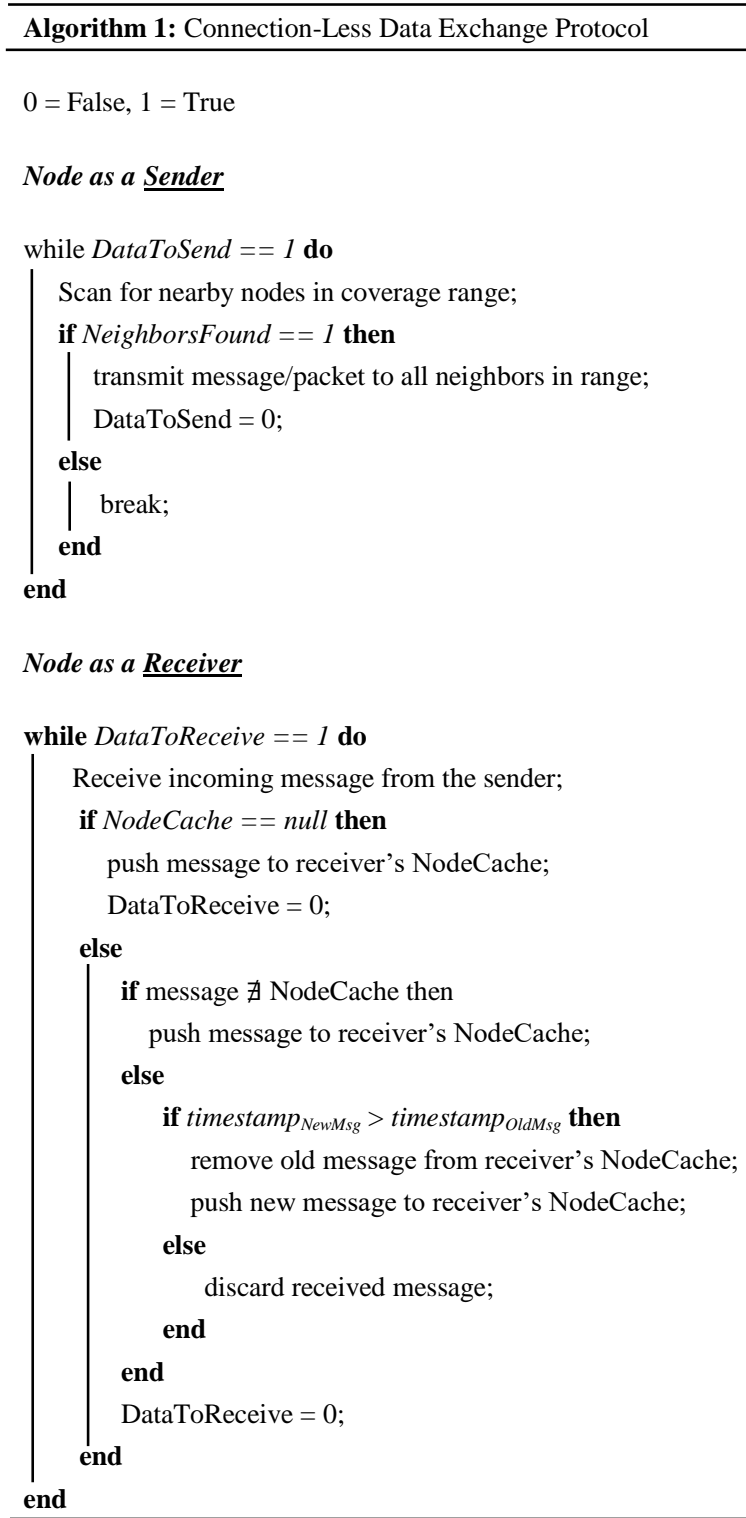

The range of the UAV has been termed as the 'coverage area' of the UAV and is defined to be the projection imposed by the UAV's antenna on the ground while flying at a constant height. The height is the altitude of the UAV whereas the coverage area is a circular area of radius 'Radius' as shown in Fig. 6.

The radius and coverage area of the UAV are defined by Equation 1 and 2, respectively. Throughout the evaluation, the range of the UAV is achieved by controlling the projection angle made by the UAV's antenna with the ground.

$\Phi=\frac{\text { Projection Angle }}{2}=\frac{\theta}{2}$

\section{Radius $=$ Altitude $\times \tan \Phi$}

Coverage Area $=\pi \times(\text { Altitude } \times \tan (\Phi))^{2}$

Waypoints have been placed towards the edges of the field in such a way that optimum coverage is achieved by the UAV while on its flight between them. The coordinates for placing the waypoints have been set using equations 3, 4, 5 and 6 .

$$
\begin{aligned}
& W P 1=\left(\frac{\text { Radius }}{\sqrt{N d a}} \times i, \frac{\text { Radius }}{\sqrt{N d a}} \times i\right) \\
& W P 2=\left(i-\frac{\text { Radius }}{\sqrt{N d a}} \times i, \frac{\text { Radius }}{\sqrt{N d a}} \times i\right) \\
& W P 3=\left(i-\frac{\text { Radius }}{\sqrt{N d a}} \times i, i-\frac{\text { Radius }}{\sqrt{N d a}} \times i\right) \\
& W P 4=\left(\frac{\text { Radius }}{\sqrt{N d a}} \times i, i-\frac{\text { Radius }}{\sqrt{N d a}} \times i\right)
\end{aligned}
$$

Where 'Radius' is the coverage range of the UAV (m), ' $N d a$ ' is the node distribution area $(\mathrm{m})$ and ' $i$ ' is equivalent to ' $\mathrm{n}$ ' from the ' $\mathrm{n} \times \mathrm{n}$ ' square matrix used in the code of the simulator for the node distribution area.

The flight pattern or the pattern of area coverage by the UAV can be understood from Fig. 7 which shows the series of areas covered by a UAV starting at time ' $n$ ' and increasing by ' $x$ ' (one second in our simulation) at each interval.

These concepts are realised in a simulation environment and then evaluated. Details of the experimental setup, the results and an assessment of their significance are provided for the different caching and non-caching variants in Section 4.

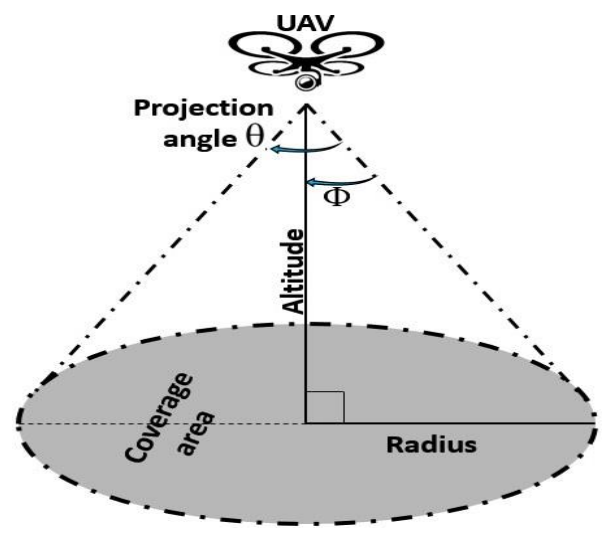

Fig. 6. Coverage Area of the UAV.

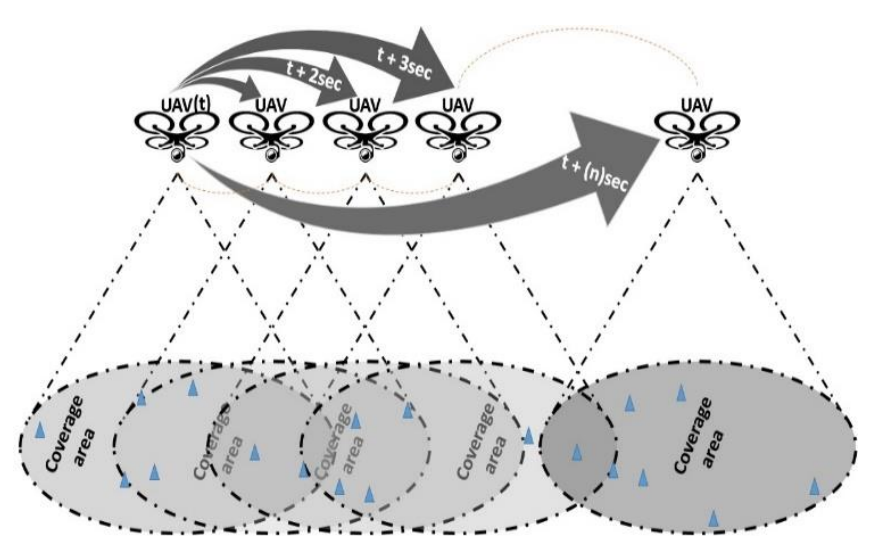

Fig. 7. Pattern of Area Coverage by the UAV. 


\section{Simulation EVALUATION}

To evaluate the benefit of caching, a bespoke JAVA run time event driven simulator was created. Several experiments were conducted testing comparing the various caching and non-caching variants presented in Section 3 under different conditions. The aim was to assess the efficacy of using intranode caching on mobile wireless sensor nodes for animal monitoring.

Three different control parameters are considered, namely: node density, node coverage range and UAV coverage range. In each scenario 10 simulations have been run varying the initial node dispersion in the distribution area. The nodes are deployed with varying speeds but the speed of the UAV remains constant throughout all simulations. The speed of the UAV has been set according to some commercial copters available in the market whereas the speed of the nodes have been set assuming the animals roam under normal conditions without stress. The range of the nodes is dependent on the antenna they host and has been set according to [43] considering not just the basic motes currently available but advanced ones with a higher ranges [44]. We have tried to mimic large herbivores such as elephants which according to [45], [46] employ the Levy walk model, normally move with speeds in access of $2 \mathrm{mps}$, have a minimum home range of ten square kilometres [47], and can exist individually or in groups between 8-100 elephants in an area [48]. The mobility model is thus a stochastic Levy model where a node determines its next step according to a Markov based decision process, whereas the UAV follows a fixed linear flight path between multiple waypoints set within the distribution area. More details of the simulation parameters are provided in Tables II and III.

TABLE II. SIMULATION AND UAV CHARACTERISTICS

\begin{tabular}{|c|c|}
\hline \multicolumn{2}{|l|}{ Simulation Parameters } \\
\hline Simulations & 10 per scenario \\
\hline Node distribution area (Nda) & $10 \mathrm{~km} \times 10 \mathrm{~km}$ \\
\hline Simulation duration & UAV's roundtrip $(\approx 45 \mathrm{mins})$ \\
\hline UAV altitude & Constant $(100 \mathrm{~m})$ \\
\hline UAV speed & Constant $(\approx 15 \mathrm{~m} / \mathrm{s})$ \\
\hline UAV coverage radius & Variable $(80 m-720 m)$ \\
\hline UAV mobility & Linear (waypoint-waypoint) \\
\hline Transfer time (UAV $\leftrightarrow$ node) & Instantaneous \\
\hline Node Density $\left(\mathrm{KM}^{-2}\right)$ & $1-7$ \\
\hline
\end{tabular}

TABLE III. NODE ATtRIBUTES

\begin{tabular}{|l|l|}
\hline Node attributes & Mobile \\
\hline Node type & Variable $(\approx 2 \mathrm{~m} / \mathrm{s}-\approx 5 \mathrm{~m} / \mathrm{s})$ \\
\hline Node speed & Variable $(80 \mathrm{~m}-720 \mathrm{~m})$ \\
\hline Node coverage & Levy model \\
\hline Mobility & Unlimited \\
\hline Cache size & No Constraint \\
\hline Energy &
\end{tabular}

In each simulation, the UAV completes one flight starting and ending at the initial waypoint / take-off point. The field size, as pointed out previously is based on reported minimum home range of elephants however, other authors have also used such values [20] in similar scenarios. Node densities are considered using information presented in [49], [50] in addition to the values used for similar work in [51]. For the sake of simplicity, it is also assumed that the data transfer time between the nodes and the UAV is instantaneous. Specific UAV attributes can be seen in Table II which has been set considering current domestic and commercial UAV characteristics. It is assumed that the UAV is not resourceconstrained compared to the sensor nodes, thus it can house an antenna potentially providing greater coverage than the sensing nodes. The node attributes can be seen in Table III. For the sake of this research, it is assumed that the nodes are not constrained by data storage or battery life; however, the coverage range has been selected based on motes available in the market and discussed in [52]. Movement speeds of nodes have been selected based on the stress free movement speed of the animals under consideration however they also match the parameters used for related work in [53].

Results are being presented using GNUPLOT version 5.2 patch 6.

Fig. 8 to Fig. 23 show the percentage of nodes encountered by the UAV and the Waypoints with and without the intermediate caching enabled between the nodes. The results are produced by varying certain perimeters to understand how it would affect the caching efficiency. All four variants of the two phases discussed in Section 3 are included.

In Fig. 8 , the coverage of the UAV is set to $720 \mathrm{~m}$ with a node density of 7 per square kilometres while varying the coverage radius of the nodes. Results show the $95 \%$ confidence intervals. Fig. 9 shows the percentage of nodes encountered by the UAV on its round trip when the coverage radius of the nodes is set to $720 \mathrm{~m}$, again with 7 nodes per square kilometres, while varying the coverage radius of the UAV. Fig. 10 illustrates that caching performance is consistent when the nodes are moving at lower speeds (equivalent to our animals in consideration). This means that for animals with movement speeds between $\approx 1.9 \mathrm{mps}$ to $\approx 5 \mathrm{mps}$, the percentage of nodes captured by the UAV is more dependent on the parameters of the UAV and the nodes rather than the movement speeds of the sensors. Fig. 11 shows the percentage of nodes cached by the UAV on its round trip when the density of the deployed nodes in the distribution area is increased from 1 to 7 per square kilometre whilst keeping the UAV and node coverage range constant. Fig. 12, Fig. 13 and Fig. 14 is a repetition of the same scenario however; the UAV and node coverage ranges have been brought down to 560m. Fig. 15, Fig. 16 and Fig. 17 repeats this with the coverage ranges of the UAV and the nodes to be set to $400 \mathrm{~m}$. Fig. 18, Fig. 19 and Fig. 20 drops the coverage range further down to $240 \mathrm{~m}$ followed by Fig. 21, Fig. 22 and Fig. 23 where the range has been set to $80 \mathrm{~m}$. All marking on the charts are with a $95 \%$ confidence interval. 
UAV Coverage Radius 720m, Node Density 7/ $\mathrm{Km}^{2}$

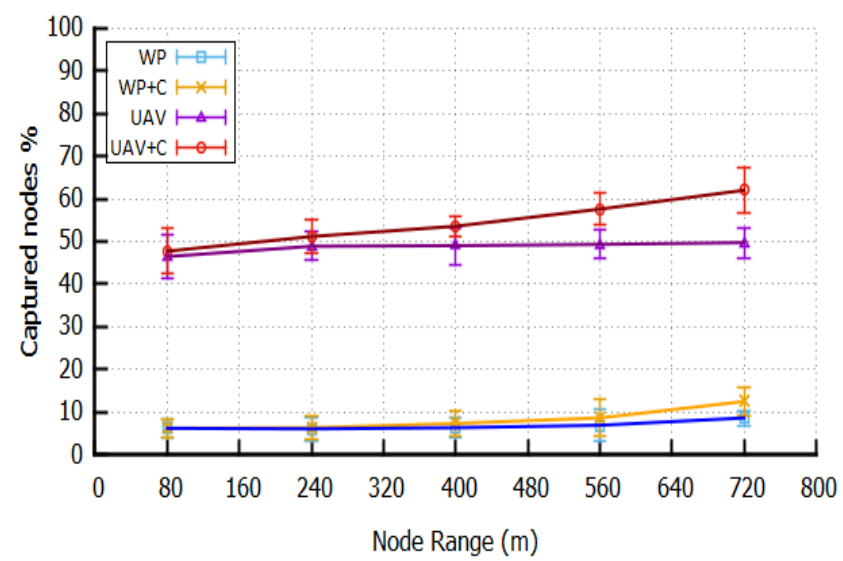

Fig. 8. Percentage of Encountered Nodes with Varying Node Coverage Range (inc. 95\% CI).

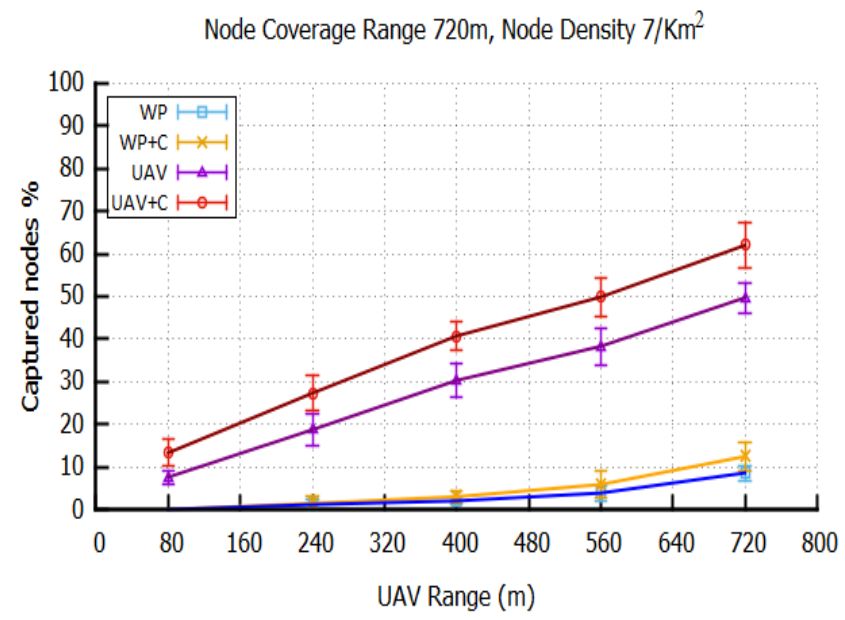

Fig. 9. Percentage of Encountered Nodes Varying UAV Coverage Range (inc. 95\% CI).

UAV Coverage Radius 720m,Node Range 400m, Node Density 7/Km²

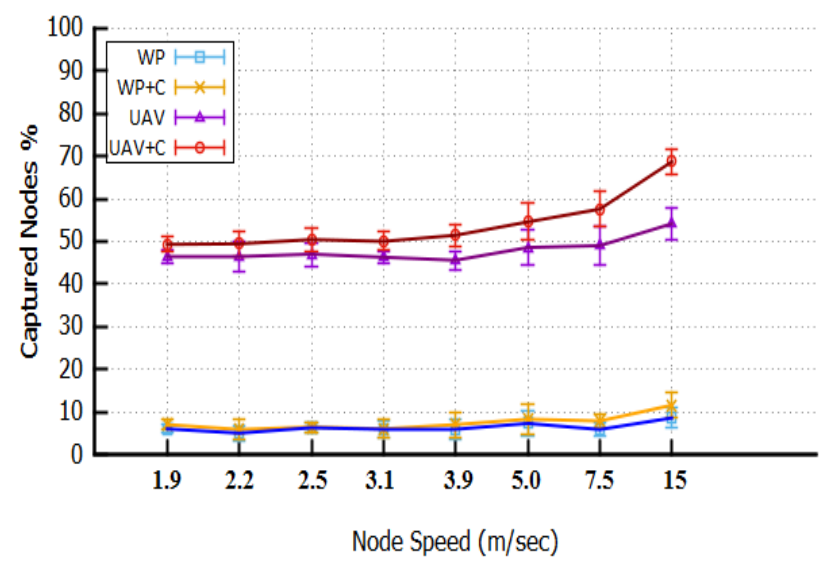

Fig. 10. Encountered Nodes \% with Varying Deployed Node Speed in Node Distribution Area (inc 95\% CI).
UAV and Node Coverage Range $720 \mathrm{~m}$

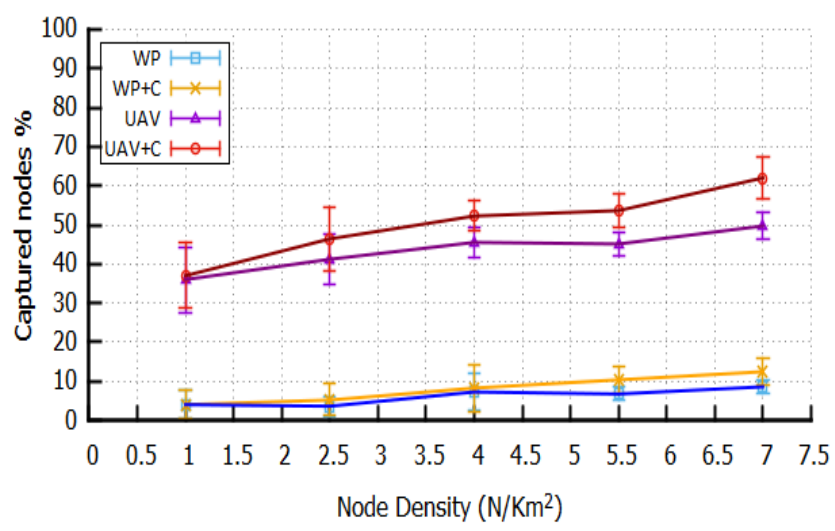

Fig. 11. Encountered Nodes \% with Varying Deployed Node Density in Node Distribution Area (inc 95\% CI) with Range $\approx 0.7 \mathrm{~km}$.

UAV Coverage Radius 560m, Node Density 7/Km2

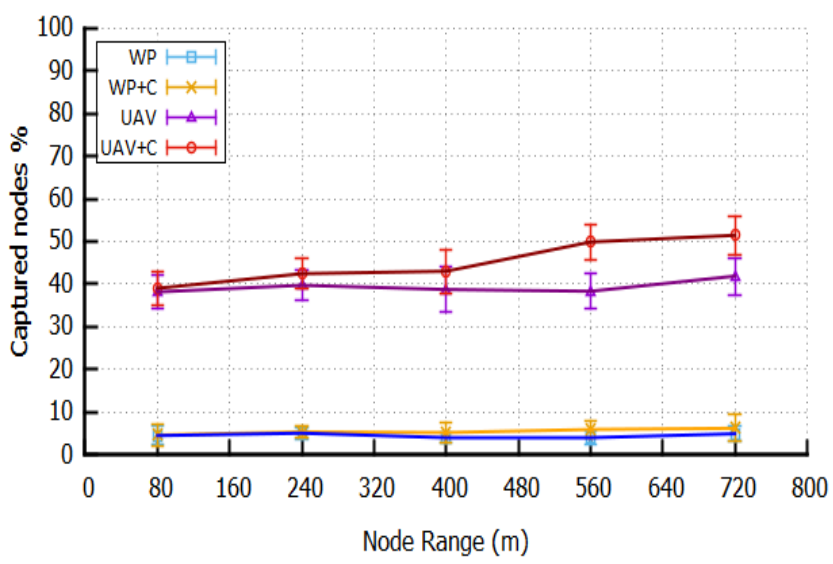

Fig. 12. Percentage of Encountered Nodes with Varying Node Coverage Range (inc. 95\% CI).

Node Coverage Range 560m, Node Density 7/Km2

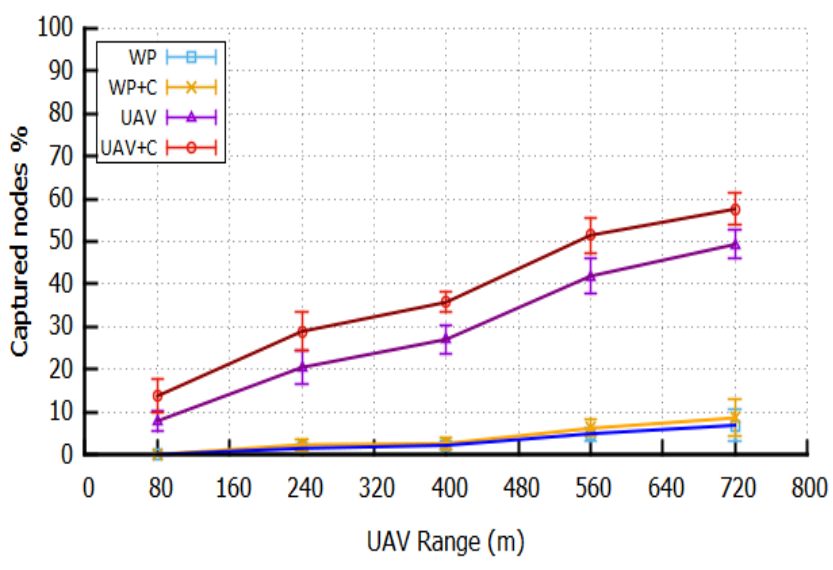

Fig. 13. Percentage of Encountered Nodes Varying UAV Coverage Range (inc. 95\% CI). 
UAV Node Coverage Range 560m

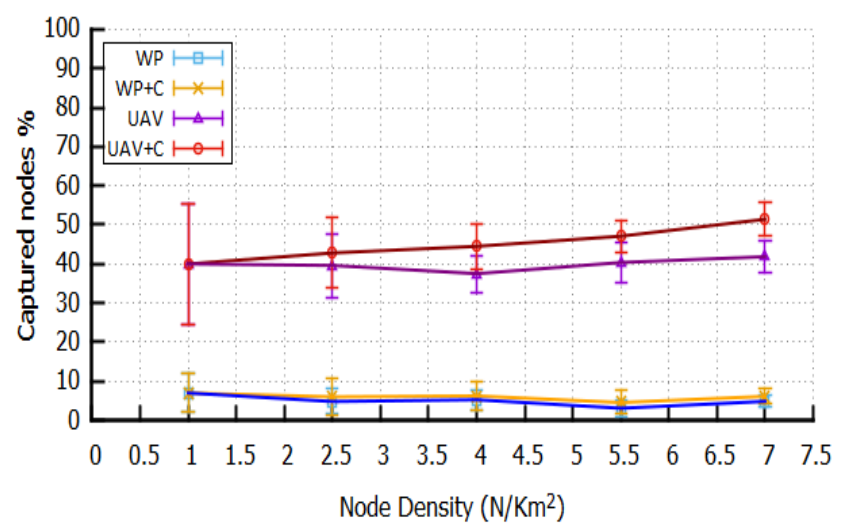

Fig. 14. Encountered Nodes \% with Varying Deployed Node Density in Node Distribution Area (inc 95\% CI) with Range $\approx 0.5 \mathrm{~km}$.

UAV Coverage Radius 400m, Node Density 7/Km2

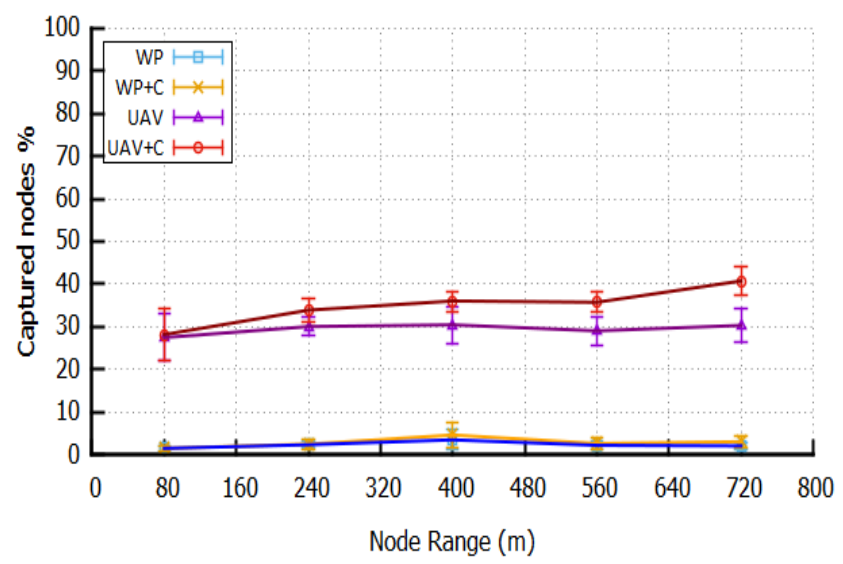

Fig. 15. Percentage of Encountered Nodes Varying Node Coverage Range (inc. 95\% CI).

Node Coverage Range 400m, Node Density 7/Km2

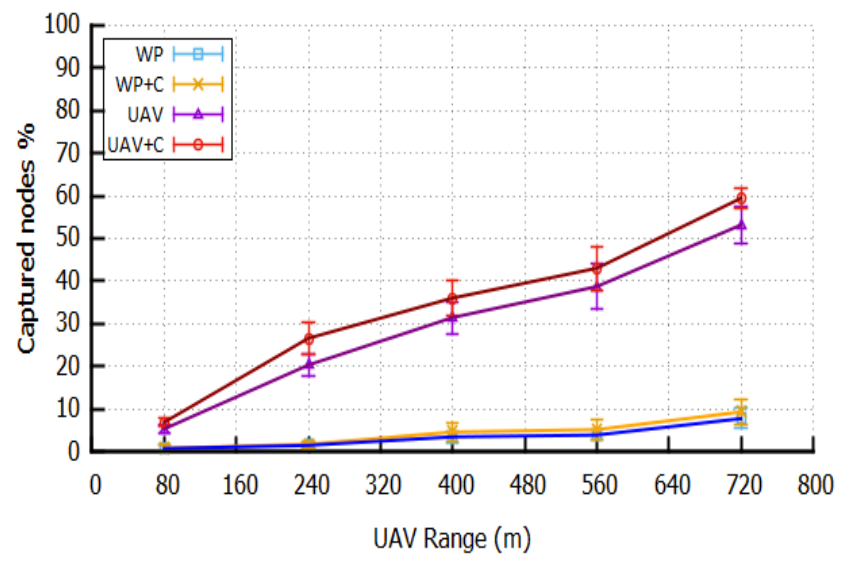

Fig. 16. Percentage of Encountered Nodes Varying UAV Coverage Range (inc. 95\% CI).
UAV and Node Coverage Range $400 \mathrm{~m}$

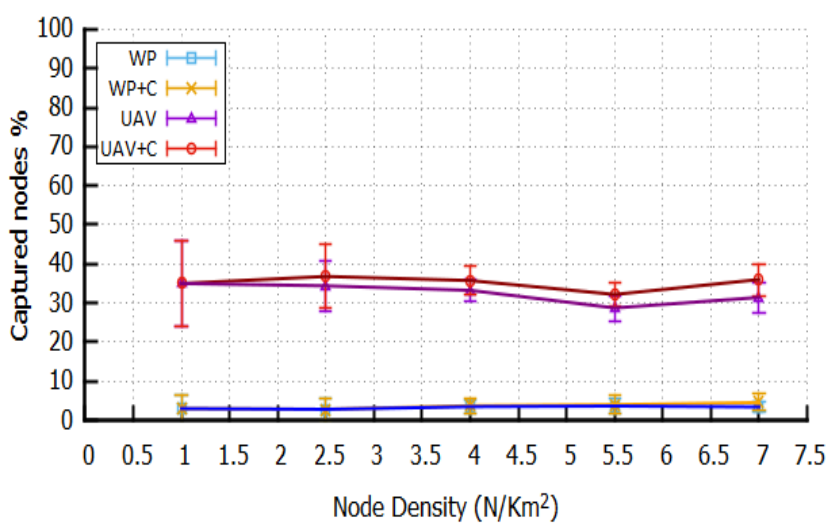

Fig. 17. Encountered Nodes \% with Varying Deployed Node Density in Node Distribution Area (inc 95\% CI) with Range $\approx 0.4 \mathrm{~km}$.

UAV Coverage Radius 240m, Node Density 7/Km2

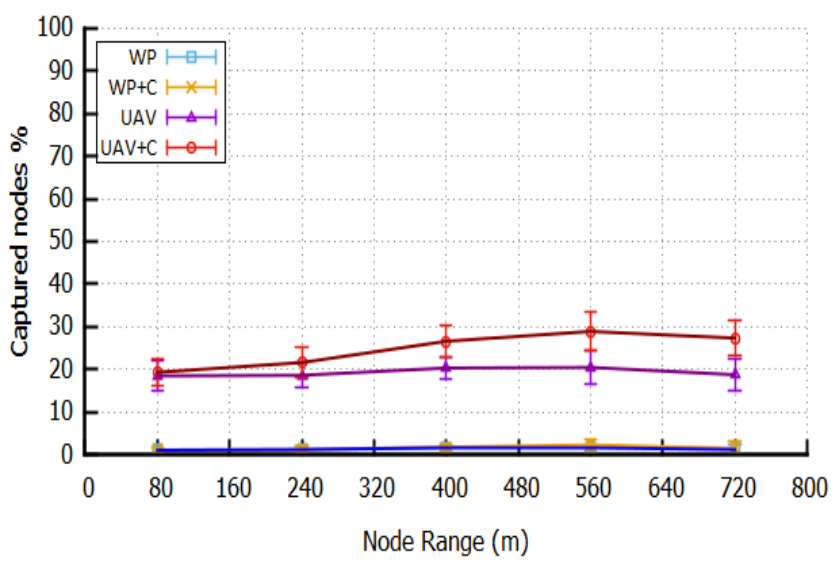

Fig. 18. Percentage of Encountered Nodes Varying node Coverage Range (inc. $95 \% \mathrm{CI}$ ).

Node Coverage Range 240m, Node Density 7/Km2

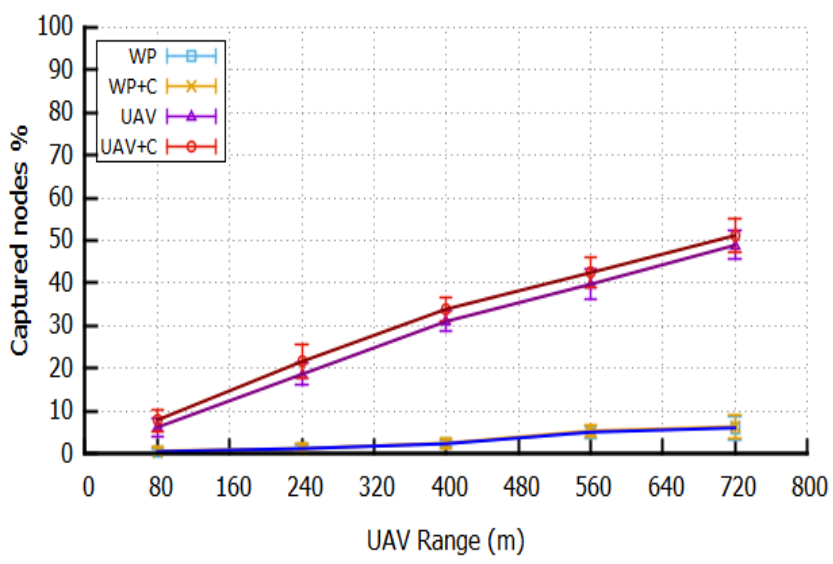

Fig. 19. Percentage of Encountered Nodes Varying UAV Coverage Range (inc. $95 \% \mathrm{CI}$ ). 
UAV and Node Coverage Range 240m

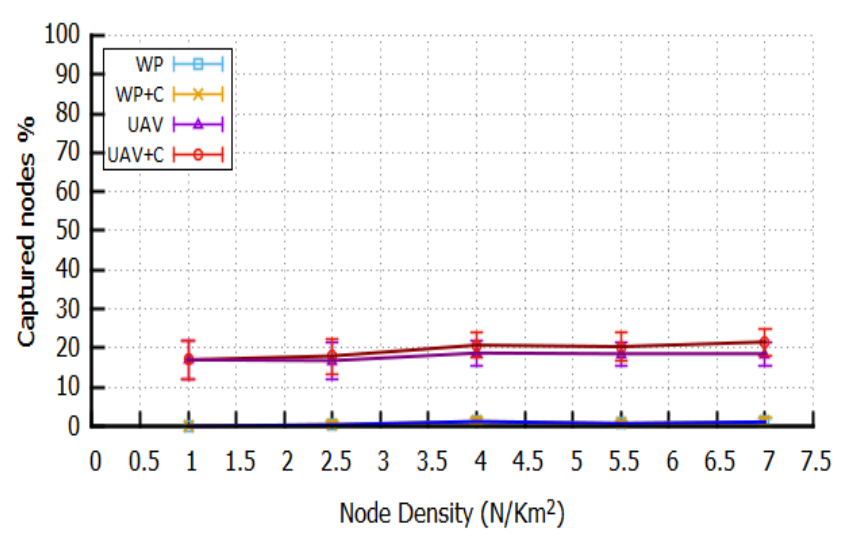

Fig. 20. Encountered Nodes \% with Varying Deployed Node Density in Node Distribution Area (inc 95\% CI) with Range $\approx 0.25 \mathrm{~km}$.

UAV Coverage Radius 80m, Node Density 7/Km2

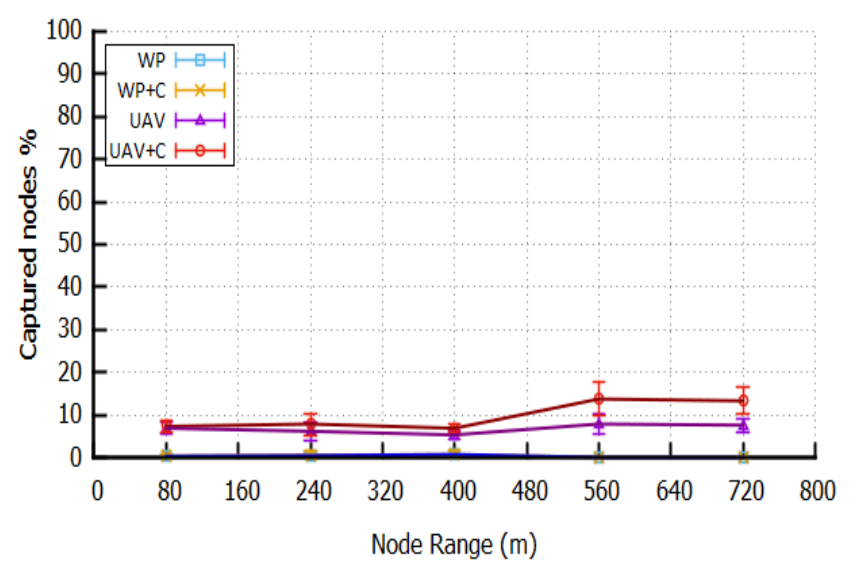

Fig. 21. Percentage of Encountered Nodes Varying Node Coverage Range (inc. $95 \% \mathrm{CI}$ ).

Node Coverage Range 80m, Node Density 7/Km2

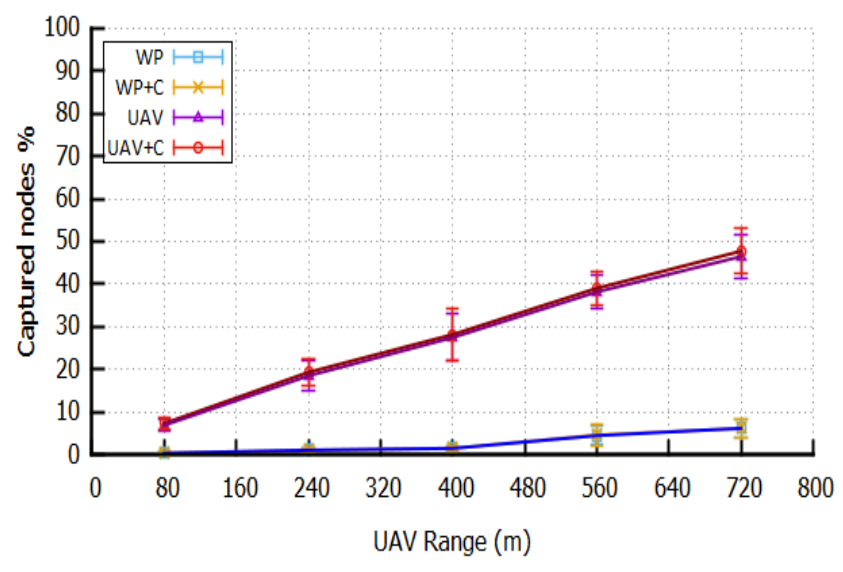

Fig. 22. Percentage of Encountered Nodes Varying UAV Coverage Range (inc. 95\% CI).
UAV and Node Coverage Range 80m

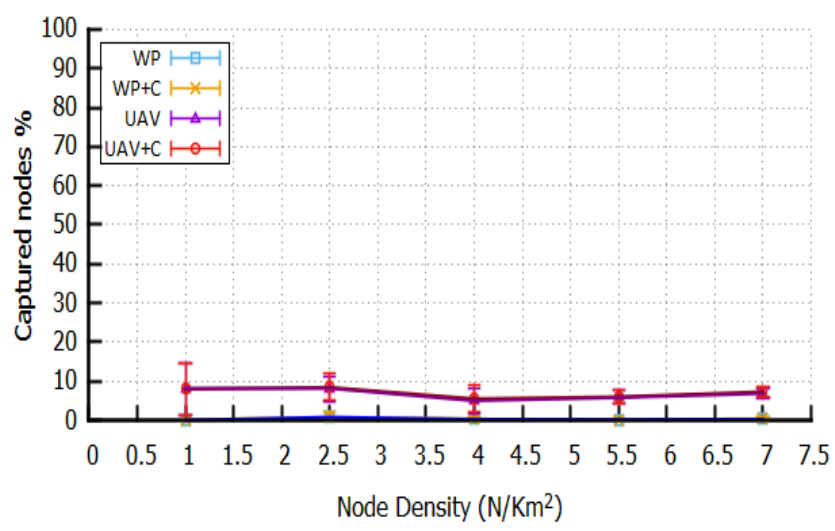

Fig. 23. Encountered Nodes \% with Varying Deployed Node Density in Node Distribution Area (inc 95\% CI) with Range $\approx 0.1 \mathrm{~km}$.

Observing from the figures, we see that the percentage of encountered nodes is highly dependent on the coverage range of the nodes with higher efficiencies when the nodes coverage radius increases. Also, the ability of the UAV to directly interact with the mobile nodes provides considerable benefit. The figures show that when the range of the nodes remains constant throughout, the improvement in the number of nodes seen by the UAV does increase but not dramatically. The percentage of nodes seen by the UAV greatly improves when the nodes are allowed to move at high speeds. This is mainly because nodes have a higher probability of interacting with and caching other nodes when they are moving faster compared to when they are moving slower and ultimately, more nodes will be picked up by the UAV. The figures also seems to suggest that for the animals in our consideration (elephants) and other similar animals, their real time movement state/speed will not have a big impact on the results considering their top speeds are not that significant and lie within the values we have used. The figures also show that for a very low deployed node count, unless the node and UAV perimeters are considered, the benefit of caching at node level is negligible and the system behaves the same with and without intra-node caching. The improvement in the percentage of nodes seen by the UAV tends to increase with the growing node density. We note again that the percentage of encountered nodes is highly dependent on the coverage range of the nodes with higher efficiencies when the nodes coverage radius increases. It shows that even when the UAV range is low, the advantages of using intermediate caching over no caching at all is quite significant. It shows that in a densely populated areas, the advantages of using intermediate caching outweigh the scenario where no caching is used. At low densities, intermediate caching and no caching yield the same results. At low node ranges, there is no advantage of embedding caching mechanisms into the deployed nodes however, for nodes with higher coverage range, a significant improvement can be seen over the previous case of no intermediate caching. The figures also seems to suggest that the advantages of intermediate caching over no intermediate caching rely more on a higher node coverage range compared to a higher UAV coverage range. At low range nodes, regardless of what the density of the environment is, 
intermediate caching yields almost no benefit. We can see that with a higher number of nodes deployed in the distribution area, the advantage of using intra-node caching is much more beneficial.

Fig. 24 and Fig. 25 demonstrated the benefits of using intermediate caching. The trend lines clearly show that in both scenarios (when the data is being collected by the UAV directly from the nodes and when it is being collected only from waypoints by the UAV), there is an improvement over the total number of nodes captured by the UAV during its trip where, a significant advantage is shown when the UAV is allowed to capture data by directly interacting with the nodes.

Our evaluation clearly shows the benefit of using intranode caching in a routing-less wireless mobile ad-hoc sensor network environment and a UAV is used as a relay agent to move data between the sensor nodes and the sink. Fig. 26 below shows the average number of nodes captured by the UAV at different node densities in the distribution area. The next section outlines our conclusions and future work.

\section{Caching Improvement Trend At the UAV}

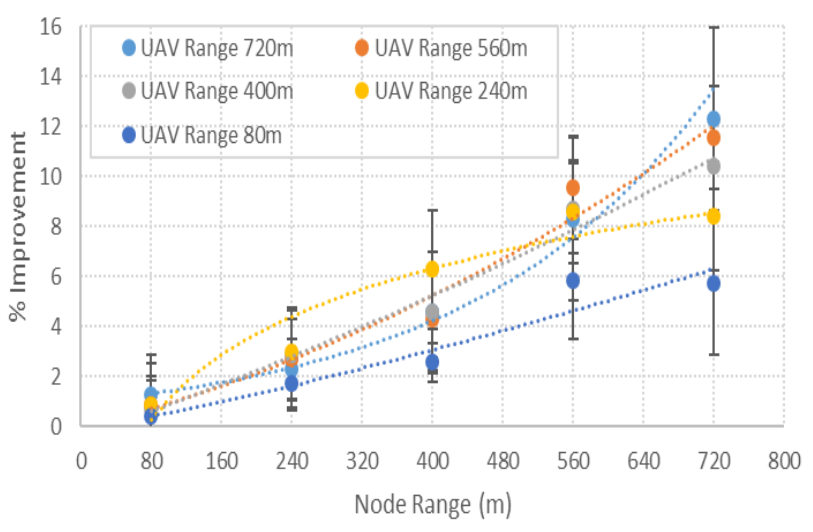

Fig. 24. Caching Improvement Trend for Nodes Captured by UAV with Intermediate Caching (inc. 95\% CI).

\section{Caching Improvement Trend At the WAYPOINT}

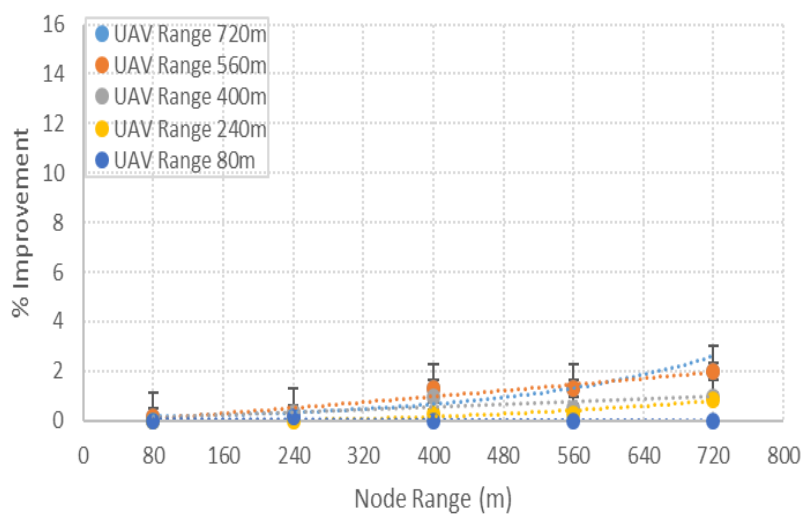

Fig. 25. Caching Improvement Trend for Nodes Captured by WAYPOINTS with Intermediate Caching (inc. 95\% CI).

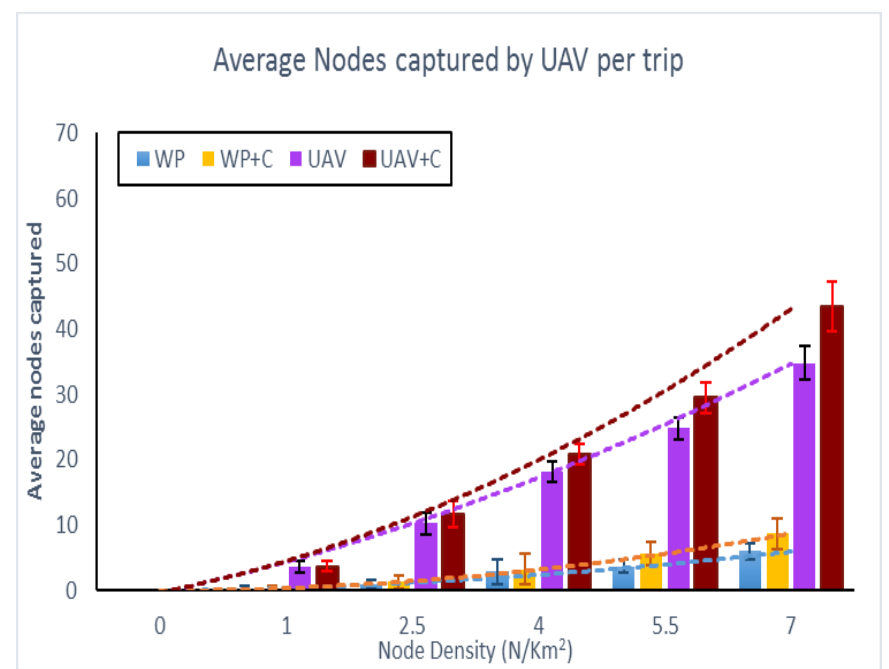

Fig. 26. Average Number of Nodes Encountered by the UAV While Increasing the Deployed Node Density (inc 95\% CI).

\section{CONClUSiONS AND FUTURE WORK}

It is clear from the results that the use of caching in a mobile ad-hoc sensor network where a UAV is used to relay information from the nodes to the sink and vice versa rather than a routing protocol yields significant benefits compared to the scenario where no caching is used. We can see from the results presented in Section 4 that even for a short duration (45mins), we were able to achieve around $12 \%$ improvement on the encountered node percentage when the UAV interacts with the nodes directly and around $4 \%$ improvement when the UAV only collects data from the waypoints. Another thing to notice here is that the UAV was allowed only one trip across the field in our experimentations however, we predict that we can further see a significant increase in the nodes seen percentage provided that the UAV makes more than one trips to collect data from the sensor nodes. In addition, the trip timings may also play a reasonable role in the percentage of nodes encountered by the UAV as we did notice in some cases that the number of nodes cached at the sensor nodes and the waypoints were higher than the nodes encountered by the UAV. The reason behind this was that the nodes and waypoints had encountered additional nodes after the UAV's visit to them hence the UAV was unable to get that information from them. In our experimentations, we chose the simplest locations for the waypoints, being around the edges of the field however, introducing no-go zones in the area and most visited zones can also have an impact on the results. Also, repositioning the waypoints to other locations based on the geography of the area where the model is to be deployed can also have an impact on the nodes encountered by the UAV on its trip. With the addition of different obstacles in the distribution area, the positioning of the waypoints can greatly affect the performance of the approach. Another interesting area to consider is selective caching with a finite cache size as opposed to our infinite caching model. Also, effects on the percentage of nodes seen by the UAV when the animals are moving rapidly are shown in Section 4; however, when the UAV moves at varying speed or the relationship between the speed of the nodes and the perimeters of the UAV can be further investigated and can yield different results as well. 
In addition to what has been discussed, there are several other parameters that we would like to check including different movement models and the use of multiple UAVs. We believe that our work opens up a very broad area of extensive research that can greatly benefit not only the field of tracking and preservation of wildlife but also, if implemented with custom parameters under different settings, can greatly assist in other areas as well including vehicular ad-hoc networks and disaster area networks as well.

\section{REFERENCES}

[1] S. Jeong, J. Ko, and J. Kim, "The effectiveness of a wireless sensor network system for landslide monitoring," IEEE Access, vol. 8, pp. 8073-8086, 2020.

[2] H. R. Galappaththi and G. T. Weerasuriya, "Implemented for Environmental Sensing," 2018 3rd Int. Conf. Inf. Technol. Res., pp. 1-6, 2018.

[3] R. Vera-Amaro, M. E. R. Angeles, and A. Luviano-Juarez, "Design and Analysis of Wireless Sensor Networks for Animal Tracking in Large Monitoring Polar Regions Using Phase-Type Distributions and Single Sensor Model," IEEE Access, vol. 7, pp. 45911-45929, 2019.

[4] T. Bensiradj and S. Moussaoui, "Strategy efficient to extend the lifetime of wireless sensor networks in a framework of hybrid sensors and vehicular networks for road safety," IET Wirel. Sens. Syst., vol. 9, no. 6, pp. 416-423, 2019.

[5] R. Singh and G. M. Asutkar, "Survey on various wireless sensor network techniques for monitoring activities of wild animals," ICIIECS 2015 - 2015 IEEE Int. Conf. Innov. Information, Embed. Commun. Syst., pp. 1-5, 2015.

[6] T. O. Olasupo, "Wireless Communication Modeling for the Deployment of Tiny IoT Devices in Rocky and Mountainous Environments," IEEE Sensors Lett., vol. 3, no. 7, pp. 1-4, 2019.

[7] "A detailed survey on Bandwidth efficient cluster based routing schemes in wireless sensor networks," no. Icssit, pp. 1250-1253, 2019.

[8] J. D. Á. V, D. L. A. S, R. P. León, and P. Sergio, "Analysis of energetic efficiency in routing protocols and sustaining quality service applied to a wireless sensor network," pp. 27-32, 2019.

[9] T. M. Behera, S. K. Mohapatra, U. C. Samal, M. S. Khan, M. Daneshmand, and A. H. Gandomi, "I-SEP: An Improved Routing Protocol for Heterogeneous WSN for IoT-Based Environmental Monitoring," IEEE Internet Things J., vol. 7, no. 1, pp. 710-717, 2019.

[10] P. Juang, H. Oki, Y. Wang, M. Martonosi, L. S. Peh, and D. Rubenstein, "Energy-efficient computing for wildlife tracking: Design tradeoffs and early experiences with ZebraNet," Int. Conf. Archit. Support Program. Lang. Oper. Syst. - ASPLOS, pp. 96-107, 2002.

[11] N. Adam, C. Tapparello, M. N. Wijesundara, and W. Heinzelman, "JumboNet Elephant Tracking Using Delay-Tolerant Routing with Multiple Sinks," 2018 Int. Conf. Comput. Netw. Commun., pp. 689695, 2018.

[12] R. Kays et al., "Tracking animal location and activity with an automated radio telemetry system in a tropical rainforest," Comput. J., vol. 54, no. 12, pp. 1931-1948, Nov. 2011.

[13] V. Dyo et al., "Wildsensing: Design and deployment of a sustainable sensor network for wildlife monitoring," ACM Trans. Sens. Networks, vol. 8, no. 4, 2012.

[14] R. Kays et al., "Tracking animal location and activity with an automated radio telemetry system in a tropical rainforest," Comput. J., vol. 54, no. 12, pp. 1931-1948, 2011.

[15] E. D. Ayele, N. Meratnia, and P. J. M. Havinga, "Towards a new opportunistic iot network architecture for wildlife monitoring system," 2018 9th IFIP Int. Conf. New Technol. Mobil. Secur. NTMS 2018 Proc., vol. 2018-Janua, pp. 1-5, 2018.

[16] S. Toledo, O. Kishon, Y. Orchan, A. Shohat, and R. Nathan, "Lessons and Experiences from the Design, Implementation, and Deployment of a Wildlife Tracking System," in Proceedings - 2016 IEEE International Conference on Software Science, Technology and Engineering, SwSTE 2016, 2016, pp. 51-60.
[17] S. Blake, I. Douglas-Hamilton, and W. B. Karesh, "GPS telemetry of forest elephants in Central Africa: Results of a preliminary study," Afr. J. Ecol., vol. 39, no. 2, pp. 178-186, 2001.

[18] P. Sommer, B. Kusy, P. Valencia, R. Dungavell, and R. Jurdak, "DelayTolerant Networking for Long-Term Animal Tracking," IEEE Internet Comput., vol. 22, no. 1, pp. 62-72, 2018.

[19] J. Xu, G. Solmaz, R. Rahmatizadeh, L. Boloni, and D. Turgut, "Providing Distribution Estimation for Animal Tracking with Unmanned Aerial Vehicles," 2018 IEEE Glob. Commun. Conf. GLOBECOM 2018 - Proc., 2018.

[20] J. Xu, G. Solmaz, R. Rahmatizadeh, D. Turgut, and L. Boloni, “Animal monitoring with unmanned aerial vehicle-Aided wireless sensor networks," Proc. - Conf. Local Comput. Networks, LCN, vol. 26-29Octo, pp. 125-132, 2015.

[21] A. Fotouhi, M. Ding, and M. Hassan, "Dynamic base station repositioning to improve performance of drone small cells," 2016 IEEE Globecom Work. GC Wkshps 2016 - Proc., 2016.

[22] A. Fotouhi, M. Ding, and M. Hassan, "Dynamic base station repositioning to improve performance of drone small cells," 2016 IEEE Globecom Work. GC Wkshps 2016 - Proc., no. 0, pp. 1-13, 2016.

[23] A. Trotta et al., "BEE-DRONES: Energy-efficient Data Collection on Wake-Up Radio-based Wireless Sensor Networks," INFOCOM 2019 IEEE Conf. Comput. Commun. Work. INFOCOM WKSHPS 2019, pp. 547-553, 2019.

[24] J. Chen et al., "Efficient Data Collection in Large-Scale UAV-aided Wireless Sensor Networks," 2019 11th Int. Conf. Wirel. Commun. Signal Process. WCSP 2019, 2019.

[25] A. Arvanitaki and N. Pappas, "Modeling of a UAV-based data collection system," IEEE Int. Work. Comput. Aided Model. Des. Commun. Links Networks, CAMAD, vol. 2017-June, 2017.

[26] S. Say, H. Inata, M. E. Ernawan, Z. Pan, J. Liu, and S. Shimamoto, "Partnership and data forwarding model for data acquisition in UAVaided sensor networks," 2017 14th IEEE Annu. Consum. Commun. Netw. Conf. CCNC 2017, pp. 933-938, 2017.

[27] X. Ma, R. Kacimi, and R. Dhaou, "Adaptive hybrid MAC protocols for UAV-assisted mobile sensor networks," CCNC 2018 - 2018 15th IEEE Annu. Consum. Commun. Netw. Conf., vol. 2018-Janua, pp. 1-4, 2018.

[28] J. Liu, X. Wang, B. Bai, and H. Dai, "Age-optimal trajectory planning for UAV-assisted data collection," INFOCOM 2018 - IEEE Conf. Comput. Commun. Work., pp. 553-558, 2018.

[29] A. F. Khalifeh, M. AlQudah, R. Tanash, and K. A. Darabkh, "A Simulation Study for UAV- Aided Wireless Sensor Network Utilizing ZigBee Protocol," Int. Conf. Wirel. Mob. Comput. Netw. Commun., vol. 2018-Octob, no. 1, pp. 181-184, 2018.

[30] S. Tansuriyavong, H. Koja, M. Kyan, and T. Anezaki, "The Development of Wildlife Tracking System Using Mobile Phone Communication Network and Drone," 2018 Int. Conf. Intell. Informatics Biomed. Sci. ICIIBMS 2018, vol. 3, pp. 351-354, 2018.

[31] H. Bayerlein, R. Gangula, and D. Gesbert, "Learning to Rest: A QLearning Approach to Flying Base Station Trajectory Design with Landing Spots," Conf. Rec. - Asilomar Conf. Signals, Syst. Comput., vol. 2018-Octob, pp. 724-728, 2019.

[32] R. Gangula, D. Gesbert, D. F. Külzer, and J. M. Franceschi, "A landing spot approach for enhancing the performance of UAV-Aided wireless networks," 2018 IEEE Int. Conf. Commun. Work. ICC Work. 2018 Proc., pp. 1-6, 2018.

[33] G. Wang, S. Lee, B. Lee, J. Young, and A. Electronics, "Secure and Efficient Cluster Head Election in a UAV-aided Wireless Sensor Network," pp. 42-49, 2019.

[34] A. M. Reynolds, "Current status and future directions of Levy walk research," Biol. Open, vol. 7, no. 1, pp. 1-6, 2018.

[35] M. E. Wosniack, M. C. Santos, E. P. Raposo, G. M. Viswanathan, and M. G. E. da Luz, The evolutionary origins of Lévy walk foraging, vol. 13, no. 10. 2017.

[36] A. O. Gautestad, "Brownian motion or Lévy walk? Stepping towards an extended statistical mechanics for animal locomotion," J. R. Soc. Interface, vol. 9, no. 74, pp. 2332-2340, 2012. 
[37] "Tikalon Blog by Dev Gualtieri." [Online]. Available: http://tikalon.com/blog/blog.php?article=2011/Levy_flight. [Accessed: 20-Apr-2020].

[38] Emanuel Deutschmann, "The Spatial Structure of Transnational Human Activity - Emanuel Deutschmann." [Online]. Available: https://www.emanueldeutschmann.net/home/new-working-paperavailable-online. [Accessed: 19-Feb-2020].

[39] D. Boyer, "What future for Lévy walks in animal movement research? Comment on "Liberating Lévy walk research from the shackles of optimal foraging', by A.M. Reynolds.," Phys. Life Rev., vol. 14, pp. 87$89,2015$.

[40] N. E. Humphries, "Why Lévy Foraging does not need to be 'unshackled' from Optimal Foraging Theory. Comment on "Liberating Lévy walk research from the shackles of optimal foraging' by A.M. Reynolds," Phys. Life Rev., vol. 14, pp. 102-104, 2015.

[41] N. Kumbhare, A. Rao, C. Gniady, W. Fink, and J. Rozenblit, "Waypoint-to-waypoint energy-efficient path planning for multicopters," IEEE Aerosp. Conf. Proc., pp. 1-11, 2017.

[42] M. T. S. Ibrahim, S. V. Ragavan, and S. G. Ponnambalam, "Way point based deliberative path planner for navigation," IEEE/ASME Int. Conf. Adv. Intell. Mechatronics, AIM, pp. 881-886, 2009.

[43] K. Akash and P. Edreena, "Survey on nodes used in wireless sensor networks," Int. J. Pharm. Technol., vol. 8, no. 4, pp. 21272-21278, 2016.

[44] S. Light, "XBee S1," pp. 1-3, 2016.
[45] X. Dai, G. Shannon, R. Slotow, B. Page, and K. J. Duffy, "ShortDuration Daytime Movements of a Cow Herd of African Elephants," J. Mammal., vol. 88, no. 1, pp. 151-157, 2007.

[46] K. M. Njoki, "Elephant Foraging Behaviour: Application of Levy Flights in Geo-information Science and Remote Sensing," 2009.

[47] N. Shadrack, M. O. Moses, M. Joseph, M. Shadrack, N. Steve, and I. James, "Home range sizes and space use of African elephants (Loxodonta africana) in the Southern Kenya and Northern Tanzania borderland landscape," Int. J. Biodivers. Conserv., vol. 9, no. 1, pp. 926, 2017.

[48] "Basic Facts About Elephants - Global Sanctuary For Elephants." [Online]. Available: https://globalelephants.org/the-basics/. [Accessed: 18-Aug-2020].

[49] K. Kangwana, Studying Elephants. 1996.

[50] S. R. Loarie, R. J. V. Aarde, and S. L. Pimm, "Fences and artificial water affect African savannah elephant movement patterns," Biol. Conserv., vol. 142, no. 12, pp. 3086-3098, 2009.

[51] M. Dong, K. Ota, M. Lin, Z. Tang, S. Du, and H. Zhu, "UAV-assisted data gathering in wireless sensor networks," J. Supercomput., vol. 70, no. 3, pp. 1142-1155, 2014.

[52] R. P. Narayanan, T. V. Sarath, and V. V. Vineeth, "Survey on Motes Used in Wireless Sensor Networks: Performance \&amp; Parametric Analysis," Wirel. Sens. Netw., vol. 08, no. 04, pp. 51-60, 2016.

[53] M. B. Kim, “Doctorat De L ' Université De Toulouse Titre :," no. May, pp. 1-190, 2019. 